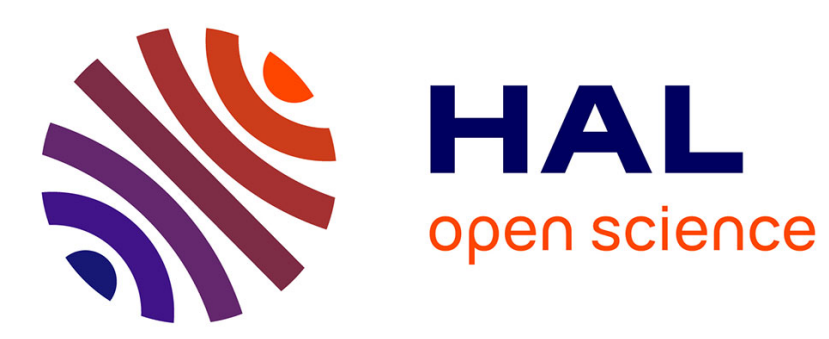

\title{
Analyse morale de la spéculation
}

Caroline Marie-Jeanne

\section{- To cite this version:}

Caroline Marie-Jeanne. Analyse morale de la spéculation. Revue d'économie financière, 2018, 131, pp.271-284. 10.3917/ecofi.131.0269 . hal-02020592

\section{HAL Id: hal-02020592 \\ https://hal.science/hal-02020592}

Submitted on 5 Jun 2020

HAL is a multi-disciplinary open access archive for the deposit and dissemination of scientific research documents, whether they are published or not. The documents may come from teaching and research institutions in France or abroad, or from public or private research centers.
L'archive ouverte pluridisciplinaire HAL, est destinée au dépôt et à la diffusion de documents scientifiques de niveau recherche, publiés ou non, émanant des établissements d'enseignement et de recherche français ou étrangers, des laboratoires publics ou privés. 


\title{
Analyse morale de la spéculation
}

\author{
Caroline Marie-Jeanne
}

\begin{abstract}
Résumé
Cet article analyse la moralité de la spéculation à la lumière des textes philosophiques depuis I'Antiquité, dans le but d'apporter des pistes de réflexion sur notre système actuel. La détermination morale d'un acte dépend de l'objet, des circonstances, de l'intention et des conséquences. Nous étudions donc les liens de causalité entre le besoin, le prix et la spéculation. Pour les philosophes, la pénurie, le gain par nécessité, l'utilité du bien et le risque encouru justifient moralement la spéculation et le profit qui en résulte. Nous mettons en exergue une inversion croissante des causes et des conséquences dans la pratique, ce qui remet en question la moralité de l'acte. La spéculation dévoyée devient une fin en soi, déconnectée de l'économie réelle et potentiellement source de risque systémique pour la collectivité.
\end{abstract}

\section{Moral analysis of speculation}

\begin{abstract}
This article analyzes the morality of speculation, in light of philosophical texts since antiquity. We aim at providing another approach for our current system. The moral determination of an act depends on the object, the circumstances, the intention and consequences. For this purpose, we study the causal links between need, price and speculation. For philosophers, the shortage, the gain by necessity, the utility of the good and the risk incurred morally justify speculation and the resulting profit. We show that, in practice, causes and consequences are often more and more reversed. The morality of the act is therefore in question. Disconnected from real economy, speculation becomes an end in itself and is potentially a source of systemic risk to the community.
\end{abstract}

Classification JEL : N23, B11, G11. 
Peut-on parler de "spéculation morale " ? L'oxymore apparent ne cache-t-il pas plusieurs manières d'appréhender le problème selon les époques et les courants de pensée ? La question de la spéculation est en effet à la fois transhistorique et interdisciplinaire. Dans le monde académique, elle suscite notamment l'intérêt des historiens, des juristes ${ }^{1}$, des économistes ${ }^{2}$ et des sociologues.

A l'origine, le terme speculatio, défini dans le dictionnaire latin Gaffiot par " espionnage ", " rapport d'un espion ", est aussi utilisé au figuré pour désigner la contemplation, l'étude, la recherche théorique. Les "speculatores" dans l'armée romaine ont la fonction d'éclaireurs, ils marchent et voient loin devant. La notion n'a alors aucune connotation péjorative dans l'usage commun, bien au contraire. C'est au milieu du XVIII ${ }^{\text {ème }}$ siècle que le mot "spéculation » prend une acception financière. II désigne aujourd'hui, selon le dictionnaire Larousse, l'«opération consistant à acheter un bien en vue de réaliser un bénéfice de sa revente ultérieure ».

La bidimensionnalité de la spéculation ne laisse pas de poser problème. Pour les uns, elle favorise la liquidité, les prix d'équilibre, le transfert des risques; pour les autres, elle est illégitime du fait de sa déconnection avec l'économie réelle, elle crée des déséquilibres, des bulles spéculatives, de la volatilité, comme le souligne Mancel (2010) dans son rapport. "Toujours en éveil, inépuisable dans ses ressources, méfiante dans la prospérité, intrépide dans les revers, elle avise, conçoit, raisonne ", selon Proudhon (1857) qui critique aussi avec véhémence ses abus : "l'intrigue, l'escroquerie, la concussion ». Cela conduit nécessairement à s'interroger sur la moralité de la spéculation.

Le regard historique paraît nécessaire pour la compréhension de ces phénomènes. II est en effet frappant de constater que l'étude du passé montre des ressemblances indéniables quant au fond des choses sur la continuité des faits et des institutions. Dès l'antiquité, Aristote, dans La Politique (livre I, chapitre 4), conseille avec humour à ceux qui s'intéressent au sujet de la richesse de " recueillir les traditions répandues sur les moyens qui ont conduit quelques personnes à la fortune ". II relate notamment le cas de Thalès de Milet qui avait prévu une récolte d'olives abondante, grâce à ses connaissances en astronomie. L'observateur grec utilisa alors judicieusement l'information privilégiée détenue, acheta une option d'achat en versant une prime, des " arrhes pour la location de tous les pressoirs de Milet et de Chios ». Puis il exerça l'option, loua les pressoirs et bénéficia de l'effet de levier.

Cependant, la période la plus frappante pour le développement de la finance est l'époque médiévale. Le commerce devient l'une des grandes sources de la richesse en Occident. Les Vénitiens en retirent beaucoup de profits et s'arrogent le titre orgueilleux de "Seigneurs de l'or de la chrétienté ". Le $\mathrm{XIII}^{\mathrm{ème}}$ siècle marque une véritable révolution dans I'histoire économique et tous les historiens modernes y voient l'origine de la technique bancaire développée par les Italiens avec notamment l'escompte, les comptes courants, les virements, la création des banques de dépôt et l'assurance terrestre et maritime. Les banquiers florentins et vénitiens développent les opérations spéculatives sur l'or et l'argent, et la lettre de change permet de profiter des arbitrages de taux d'intérêt ou de taux de change entre les différentes places.

Dès lors se pose avec urgence aux philosophes le problème de la moralité du commerce et de la finance. Todeschini (2008 et 2017) montre ainsi comment, à cette époque, la place essentielle tenue dans la morale chrétienne par la notion de pauvreté impose un examen rigoureux des fonctionnements de marché, aboutissant à une analyse sans cesse plus précise des comportements économiques. Dans ce contexte, la Somme théologique de Thomas d'Aquin, écrite entre 1266 et 
1273, a un caractère central et précurseur. Le philosophe n'emploie pas le terme de spéculation dans son sens financier, mais la notion est présente dans ses études, dans lesquelles il analyse très finement les dangers constatés dans l'expérience sociale de son temps.

À la lumière des principes moraux développés par les philosophes depuis l'Antiquité, notre objectif est de proposer des pistes de réflexion pour la finance moderne. Pour qu'un acte soit moral, il faut trois conditions : l'objet, les circonstances et, selon l'approche philosophique, les conséquences ou l'intention. L'objet, c'est-à-dire la spéculation avec l'opération d'achat suivie d'une revente, est un acte en soi moralement indifférent. Les circonstances peuvent varier. Afin de déterminer la moralité de la spéculation, cet article s'intéresse donc à l'analyse de ses causes et de ses conséquences, à travers la notion de besoin, dans une première partie, puis à travers la notion de prix, dans une deuxième partie. Nous mettons en exergue, dans une troisième partie, une inversion croissante des causes et des conséquences dans la pratique, ce qui remet en question la moralité de l'acte. La spéculation dévoyée devient une fin en soi, déconnectée de l'économie réelle et potentiellement source de pénurie et de risque systémique pour la collectivité.

\section{ANALYSE DE LA CAUSALITÉ ENTRE BESOIN ET SPÉCULATION}

Pour déterminer la moralité de la spéculation, l'analyse de ses causes et de ses conséquences est nécessaire, notamment par rapport à la notion de besoin de marchandises, au sens de pénurie, et de besoin d'argent, au sens de gain. Ces interactions sont étudiées à travers l'histoire.

\section{Sous l'angle de la pénurie}

Il résulte de l'observation historique des faits que la pénurie est la cause première de la spéculation: la rareté due aux mauvaises récoltes, au climat ou aux conflits armés fait augmenter les prix des produits alimentaires. Or Garnsey (1996) évalue qu'à Rome, entre 509 et 384 avant J.-C., au moins une année sur neuf, et entre 123 et 50 avant J.-C., une année sur cinq, des pénuries surviennent causées par les guerres, les désordres civils, les épidémies en tout genre ou les aléas climatiques.

Pour limiter la famine, les États cherchent à créer des lois et des instances de régulation. À Athènes, une dîme, en nature, sur l'orge et le blé cultivés, est versée au sanctuaire de Déméter. Cette réserve peut être vendue, selon une inscription de 329-328 avant J.-C., année de pénurie, à un prix inférieur à celui du marché. En Grèce ou à Rome, l'État intervient également directement dans l'approvisionnement en céréales, peut constituer des réserves et les mettre à la disposition des citoyens, gratuitement ou à prix abordable lors des pénuries, diminuant ainsi les effets d'une mauvaise récolte et les effets de la spéculation.

Sur le plan moral, limiter la famine par l'importation de marchandises est une intention bonne. Mais est-il permis de vendre des denrées en profitant de la pénurie pour élever les prix ? Thomas d'Aquin sort de cette épineuse question avec une grande habileté. Le marchand témoignerait certes d'une plus grande vertu en avertissant la population de la venue imminente d'un approvisionnement suffisant, mais il n'agit pas contre la justice en observant le prix du marché tel qu'il le trouve au 
moment de la vente de ses grains. En bref, il n'est pas coupable car le prix n'est pas supérieur à ce que vaut la chose conformément au cours du marché au moment précis de la vente. En outre, refuser de manière radicale la possibilité qu'apporte la spéculation de mettre fin à une disette, n'estce pas compromettre les chances de sortir de la famine ? En revanche, la doctrine est très claire et va dans le même sens que la loi civile : créer une pénurie en accaparant les denrées est répréhensible.

\section{Sous l'angle du gain}

La spéculation est clairement la cause du gain, puisqu'elle consiste à acheter un bien en vue de réaliser un bénéfice de sa revente ultérieure. L'objectif ici est donc de déterminer l'intention de l'acte. Pour Aristote, l'échange nommé "chrématistique commerciale", qui a pour but l'accumulation d'argent, favorise la cupidité, laquelle n'a pas de bornes et tend à acquérir sans fin. Seul l'échange de denrées contre denrées, ou denrées contre argent, pour les nécessités de la vie est louable, légitime et juste.

«Xénophon est le seul philosophe grec qui ait reconnu sans restriction la légitimité de la propriété individuelle et glorifié la richesse ", note Espinas (1905). Pour ce contemporain de Platon, les spéculateurs sont des amoureux de leurs biens. Dans l'Économique (chapitre XX), il étudie l'art de bien gérer un grand domaine agricole et présente sa leçon dans la forme de dialogues socratiques.

"- Socrate: À t'entendre, Ischomachus, ton père avait naturellement pour l'agriculture le même goût que les marchands de blé ont pour leur commerce; et comme ces marchands-là aiment singulièrement le blé, dès qu'ils entendent parler d'un pays où il abonde, ils y naviguent, traversent la mer Egée, le Pont-Euxin, la mer de Sicile : là ils en prennent tant qu'ils peuvent, puis ils le rapportent par mer sur le vaisseau qui les porte eux-mêmes. S'ils ont besoin d'argent, ce n'est pas au hasard ni au premier endroit qu'ils déchargent le bâtiment; mais quand ils entendent parler d'un pays où le blé est à haut prix et dont les habitants sont prêts à le payer cher, ils s'y rendent et font livraison. II me semble que c'est comme cela que ton père était agriculteur passionné.

- Tu plaisantes, Socrate, répondit Ischomachus. Pour moi, je pense que ceux-là sont de vrais amateurs de maisons, qui, à mesure qu'ils en bâtissent une, la vendent pour en bâtir une autre.

- Par Jupiter, Ischomachus, répliquais-je, je suis prêt à jurer que tu as raison de croire qu'on aime naturellement ce dont on espère tirer profit. »

Mais ces négociants qui achètent des denrées pour les revendre à un prix plus élevé que celui auquel ils les ont achetées font généralement l'objet de réprobations. Au Moyen-Âge, Raymond de Pennafort, connu pour avoir rassemblé, à la demande du pape Grégoire IX, les multiples lois et décrets, ne fait d'exception qu'en faveur des artisans qui ont transformé, par leur travail manuel, la matière première acquise: le fer, le plomb ou le cuivre. "La pensée médiévale, héritière de l'antique, ne mettait pas très haut le travail, mais elle donnait sens à la souffrance nécessaire des hommes " (Lautman, 1969).

Entré dans l'ordre de Saint Dominique à l'époque de la plus grande renommée de Raymond de Pennafort, Thomas d'Aquin va plus loin et accepte clairement le négoce. Le gain « n'implique rien non plus qui soit absolument vicieux ou contraire à la vertu. Par conséquent, rien n'empêche qu'on ne le destine à une fin honnête ou nécessaire ", affirme-t-il (II ${ }^{\mathrm{a}} \|^{\mathrm{ae}}, \mathrm{Q}, 77$, art. 4). En toute chose, $c^{\prime}$ est 
la fin qui doit être recherchée. À la question « Est-il permis dans le commerce de vendre plus cher qu'on achète ? " ( $1 I^{\mathrm{a}}-\|^{\mathrm{ae}}, \mathrm{Q} 77$, art. 4), il répond que le négoce est permis " quand quelqu'un cherche à faire dans le commerce un bénéfice modéré pour soutenir sa famille ou pour secourir les pauvres, ou quand quelqu'un s'applique au commerce dans l'intérêt général, pour que sa patrie ne manque pas du nécessaire ". II juge le commerce en théologien, non en économiste. "Son but est de définir les conditions de moralité de l'action humaine, qui reçoit sa règle de la raison. À la base de toute action, en effet, il y a un jugement de valeur appréciant telle fin comme bonne et désirable, ou non. » (Spicq, 1934). Cette détermination de l'objectif est utilisée de nouveau au XIX ${ }^{\text {ème }}$ siècle par les juges afin d'appliquer le droit pénal face aux comportements spéculatifs. Pour analyser des situations techniques fort complexes, les juges ont recours à la notion de but recherché. La spéculation est donc morale en cas de pénurie ou si le besoin d'argent n'est pas mû par la cupidité. Sinon, elle n'a pas d'utilité sociale et peut même nuire à la formation des prix.

\section{ANALYSE DE LA CAUSALITÉ ENTRE PRIX ET SPÉCULATION}

L'étude des conséquences ou de l'intention de la spéculation, notamment à travers le prix, est nécessaire pour déterminer la moralité de l'acte. La notion de prix fait référence à la valeur intrinsèque ou subjective du bien, elle-même liée à l'incertitude et au risque.

\section{Sous l'angle de la valeur du bien}

La variation des prix est clairement la cause de la spéculation; elle permet de réaliser un gain. À chaque époque, les prix sont en constante évolution, au gré de l'abondance ou de la raréfaction. La question du juste prix relève donc de l'ordre moral.

Reprendre la finesse des analyses des théologiens peut être instructif pour vérifier où se joue le prix $d^{\prime}$ une chose et comment fonctionne cette norme du juste. Une réponse tentante et rapide consisterait à affirmer que la valeur d'un bien dépend de sa substance ou de sa rareté. Un blé charançonné ne vaut pas un beau blé. Le juste prix serait alors "intrinsèque ", dans la " nature » même des choses qui s'échangent et déterminable a priori de manière exogène au marché. Thomas d'Aquin accepte, au contraire, un prix variant selon les circonstances de l'échange et selon les lieux. "Le juste prix n'a pas été ponctuellement déterminé ", précise-t-il. Des études de prix menées pour le XIII ${ }^{\text {ème }}$ siècle mettent d'ailleurs en évidence des fluctuations incessantes avec des maxima et minima très marqués. Toutefois, au Moyen-Âge, les auteurs ne construisent pas une approche fondée sur le prix concurrentiel, mettant en jeu le mécanisme de l'offre et de la demande qui prévaut aujourd'hui sur le marché financier. "Ce n'est qu'assez tardivement, sous l'influence, au XVI siècle, de l'École de Salamanque et de son fondateur F. de Vitoria, que les mécanismes de marché furent explicitement pris en considération. » (Lapidus, 1986).

Mais est-il moralement permis de profiter de la nécessité d'autrui pour augmenter le prix ? Thomas d'Aquin a cerné toute la complexité de la notion de juste valeur dans son étude des relations 
synallagmatiques dans les contrats. Il affirme qu' « il est injuste et illicite en soi de vendre une chose plus qu'elle ne vaut, ou de l'acheter moins " $\left(\mathrm{II}^{\mathrm{a}}-\mathrm{II}^{\mathrm{ae}}, \mathrm{Q} 77\right.$, art. 1). II propose alors une vérité plus complète qui postule que le prix d'une chose est juste parce qu'il révèle la justice dans l'échange. Le prix juste prend figure de compensation qu'il faut accorder et qui doit être " proportionnée à l'utilité qu'on en retire " $\left(\mathrm{II}^{\mathrm{a}}-\mathrm{II}^{\mathrm{ae}}, \mathrm{Q} 77\right.$, art. 1). II dépend du jeu subtil des avantages et des préjudices que la vente et l'achat peuvent induire chez l'un ou l'autre acteur et il correspond à un équilibre tâtonnant qui ne lèserait aucune des parties contractantes. La valeur est subjective, mais, moralement, il ne s'agit pas de se prévaloir de la nécessité de l'acheteur pour augmenter le prix. " La justesse du prix ne doit pas se régler seulement sur l'objet vendu, mais encore sur la perte que le vendeur éprouve en le cédant. [...] Mais si l'on retire un grand profit de la chose d'autrui et que le vendeur, en s'en privant, ne souffre pas, il ne doit pas la vendre au-dessus de sa valeur, parce que l'avantage qui en résulte ne provient pas du vendeur, mais de la position de l'acheteur ", affirme Thomas. Ainsi, pour la détermination du prix, il avait déjà cerné la notion d'utilité comme mesure du bien-être ou de la satisfaction obtenue par l'obtention d'un bien. Ce concept sera employé, bien plus tard, dans les fonctions d'utilité en économie et par Léon Walras dans le concept d'utilité marginale.

Toute son approche se base sur la distinction entre la loi civile et la loi divine. La loi civile permet au vendeur de majorer le prix de sa marchandise et à l'acheteur de l'acheter moins cher, pourvu qu'il n'y ait pas de fraude et qu'on ne dépasse pas certaines limites. La loi divine va plus loin : elle considère comme un acte illicite le fait de ne pas observer l'égalité et la justice dans l'achat et dans la vente. Chacun doit choisir non son intérêt personnel, mais le bien commun. Thomas distingue aussi la justice commutative et l'amitié utile. La justice commutative considère principalement l'égalité des choses échangées (capacité des produits à entrer en rapport quantifiable entre eux). Mais l'amitié utile considère l'égalité de l'utilité respective, c'est pourquoi la compensation qu'il faut accorder doit être proportionnée au bienfait obtenu. À mesure que se sont effacés les idéaux médiévaux s'est imposée une problématique de justice commutative, dans laquelle prévaut la valeur d'échange. Toutefois ce concept de valeur d'usage resurgit depuis peu dans l'orbe de tout un ensemble de questionnements théoriques sur le caractère durable du développement économique et sur les craintes de ne pouvoir léguer aux générations futures les biens naturels.

\section{Sous l'angle de l'asymétrie d'information}

L'objectif est de déterminer dans quelle mesure l'information dissymétrique sur les biens peut être utilisée moralement afin d'augmenter le prix. Le premier cas, celui du mensonge ou de la fraude, est très clair : la loi civile et la loi morale l'interdisent. Concernant l'escroquerie sur l'espèce de la chose, comme du vin mélangé d'eau, ou sur la quantité de la chose, Thomas d'Aquin affirme que " si le vendeur connaît ce défaut dans l'objet qu'il vend, il commet une fraude et, par conséquent, sa vente devient illicite $»\left(\mathrm{II}^{\mathrm{a}}-\mathrm{II}^{\mathrm{ae}}, \mathrm{Q} 77\right.$, art. 2). 
Mais le second cas est plus complexe : le vendeur est-il tenu de dire les défauts de sa marchandise ? Dans son œuvre philosophique "De officiis" (partie XII du livre III), Cicéron aborde le cas d'un négociant venu d'Alexandrie à Rhodes avec une importante cargaison de blé dans un moment où, à Rhodes, on souffre d'un manque complet de vivres et d'une véritable famine. II sait que plusieurs négociants sont partis d'Alexandrie, il a vu dans sa traversée des navires chargés de blé à destination de Rhodes. "Doit-il le dire aux Rhodiens ou garder le silence pour vendre sa cargaison plus chère ? ", demande Cicéron. Sur cette question, Diogène de Babylone, grand stoïcien, soutient qu'il faut faire connaître les défauts de la marchandise, autant que l'ordonne le droit civil, tout en essayant de vendre le mieux possible. Puis Cicéron étudie le cas d'un " honnête homme qui veut vendre sa maison à cause de certains défauts qu'il sait qu'elle a et qu'ignorent les autres : elle est malsaine et on la croit salubre, on ignore que dans les chambres apparaissent des serpents, que la charpente est mauvaise et menace ruine ". L'acheteur n'a pas affiché " maison de campagne agréable et bien construite ". "Ne serait-il pas absurde qu'un crieur public annonçât par ordre du propriétaire maison insalubre à vendre ? ", demande Diogène qui ajoute : "On ne t'a pas obligé d'acheter, on ne t'y a même pas exhorté [...] tu as acheté une maison qui te plaisait. »

Cicéron voit là " l'opposition qui paraît souvent exister entre une manière profitable d'agir et une manière loyale ". Selon lui, " ni ce négociant en blé ne devait cacher quoi que ce fût aux Rhodiens, ni le propriétaire qui voulait vendre sa maison aux acheteurs ». Cacher quelque chose en pareil cas, ce n'est pas seulement ne pas dire, c'est vouloir faire ignorer à ceux qui ont intérêt à savoir afin d'en tirer profit. Ainsi la loyauté va au-delà de ce qu'exige la loi civile.

En ce qui concerne le cas des défauts, Thomas d'Aquin a une position plus souple que Cicéron et Saint-Ambroise qui préconisent de les déclarer sous peine de nullité du contrat. II reprend le cas de la pénurie de blé $\left(1 \mathrm{I}^{\mathrm{a}}-\|^{\mathrm{ae}}, \mathrm{Q} 77\right.$, art. 3) et y répond de façon limpide : " C'est à l'avenir qu'on s'attend à voir baisser le prix de la marchandise par suite de l'arrivée des marchands, ce que l'acheteur ignore. " II conclut que, par conséquent, le vendeur peut, sans blesser la justice, vendre la chose au prix du marché, sans faire connaître ce qui doit arriver. Vendre au prix du marché est donc admis et faire annoncer les défauts de la marchandise ferait « fuir les acheteurs et leur laisserait ignorer les autres qualités qui rendent cette marchandise bonne et utile ». Pour étayer son propos, Thomas distingue le cas du défaut manifeste, par exemple, un cheval borgne, pour lequel le vendeur n'est pas tenu signaler le vice caché, du cas du défaut dangereux. II n'est pas permis de vendre " une maison qui menace ruine comme une maison en bon état, ou des aliments avariés ou empoisonnés comme des aliments sains", car il est toujours illicite de fournir à autrui une occasion de danger. Le théologien là encore cherche un juste équilibre.

Cette question du signalement des défauts du bien est centrale en économie. Des mécanismes de contrôle évitent que les acheteurs pâtissent de l'asymétrie d'information et que le marché soit affecté par un phénomène de sélection adverse, mis en évidence par Akerlof (1970). Ainsi, au Moyen-Âge, en veillant au maintien de la loyauté commerciale, les guildes de commerce vont permettre de réduire les effets néfastes de l'asymétrie d'information sur la qualité des produits. " Elles excluent de leur association ou frappent d'amende celui qui est coupable d'avoir livré à un acheteur une étoffe de qualité inférieure à celle qui lui avait été montrée sur le marché, trompé sur le poids, mouillé des épices pour les rendre plus pesantes, vendu des fourrures d'occasion comme si elles étaient neuves, fourni du cuir de mouton pour du cuir de daim, etc. »(Spicq, 1934). 


\section{Sous l'angle du risque}

Le risque pris ex ante par les négociants au niveau individuel est la cause de l'augmentation des prix. Il justifie d'ailleurs le gain, pour les moralistes. Dans le négoce des marchandises, le danger du transport est bien réel à cette époque. Henri de Gand (1518) observe d'ailleurs : "Telle marchandise est vendue à vil prix dans le pays où elle abonde, qui se vendra fort cher dans un autre pays où elle est rare. Le commerçant qui a pris soin de la transporter est en droit de la vendre ce qu'elle vaut, quoiqu'il l'ait payée moins cher. " Ainsi la capacité de prévision des contrepartistes, le risque encouru et la satisfaction urgente et vitale des besoins de la communauté rendaient légitime un gain considérable : les spéculateurs pouvaient mettre fin à une disette en acceptant d'endosser l'aléa lors du commerce de denrées de première nécessité.

En supportant l'incertitude sur le marché financier, le spéculateur-assureur permet également aux agents économiques de se couvrir. La Confusion de Confusiones de Joseph de La Vega (1688) décrit de façon très poétique le fonctionnement de l'« opsie " (option similaire à un call) sur les actions de la Compagnie des Indes du marché d'Amsterdam. " Pour ce qui est des opsies, il s'agit de primes ou de quantités que l'on donne pour assurer les actions ou conquérir les avancées; elles servent de bougies pour naviguer heureux dans la prospérité et d'ancres pour naviguer en sécurité dans les orages. " Ainsi, s'il agit de manière optimale, chaque opérateur, qu'il soit acheteur ou vendeur, maximise son espérance d'utilité en couvrant un risque primitif ou en jouant sur ses anticipations. La prise en compte du risque en finance ces trente dernières années (modèle d'évaluation des actifs financiers, prime de risque, aversion au risque) n'est, en somme, qu'un développement de ces idées antérieures. Le changement réside dans l'inversion des causes et des conséquences : la spéculation devient cause de risque pour la communauté.

\section{INVERSION CROISSANTE DES CAUSES ET DES CONSÉQUENCES}

Pour les philosophes, la pénurie, le gain par nécessité, l'utilité du bien et le risque encouru justifient moralement la spéculation et le profit qui en résulte. Mais l'inversion croissante des causes et des conséquences de la spéculation par rapport au besoin et par rapport au prix remet en question la moralité de l'acte.

\section{Inversion de la causalité par rapport au besoin}

Dans l'histoire, la spéculation devient parfois la source de la pénurie ou de son aggravation. Des troubles en résultent dans le monde gréco-romain, comme en témoigne Philostrate (1862, livre I, 15) 
à propos de l'époque de l'empereur Tibère. " Les riches avaient caché le blé, dont ils faisaient trafic hors du pays. Une foule composée de personnes de tout âge était soulevée contre le gouverneur ». "Dans les sociétés grecque et romaine, on reprochait aux grands propriétaires terriens de spéculer sur le prix des aliments, ce qui entraînait à l'occasion l'intervention des autorités municipales. Ceux-ci emmagasinaient leurs céréales en attendant une pénurie et une hausse des prix avant de les proposer aux acheteurs. ", relate I'historien Nadeau (2009). La stratégie reste aujourd'hui similaire sur le marché des matières premières agricoles avec le "squeeze " qui consiste à créer une situation réelle ou artificielle de rareté du sous-jacent grâce à d'importants volumes d'achats sur le marché à terme et sur le marché physique, par exemple. De plus, deux mille ans plus tard, le blé reste un produit sensible. Ainsi, en 2007, la multiplication par deux du prix du contrat à terme sur le blé coté sur Euronext Liffe causa des émeutes de la faim dans quarante pays d'Afrique et d'Asie.

À chaque époque, les États cherchent à combattre les effets pervers de la spéculation en légiférant. Une inscription de Téos, datant de 470 av. J.-C., indique que la cité se méfiait des citoyens spéculateurs : "Si quelqu'un empêche, de quelque manière que ce soit, d'importer des céréales sur le territoire de Téos, par mer ou par voie de terre, ou qu'il pousse à la hausse le prix des céréales importées, qu'il périsse, lui-même et sa famille. » La loi d'Irni (Espagne), étudiée par Gonzalez (1986), précise aussi, à la fin du $\mathrm{I}^{\text {er }}$ siècle après J.-C. : nul dans ce municipe ne peut acheter en totalité ou accaparer ou s'entendre avec un autre ou passer un accord ou s'associer en vue de vendre une chose plus chère, ou de ne pas la vendre, ou ne pas en vendre en quantité suffisante. De même, Aristote rapporte dans La Politique (livre 1, chapitre 4) qu'un particulier en Sicile avait créé un monopole sur le fer afin de s'enrichir. "Denys en fut informé ; et tout en permettant au spéculateur d'emporter sa fortune, il l'exila de Syracuse pour avoir imaginé une opération préjudiciable aux intérêts du prince. » Cette interdiction législative de manipuler le marché se retrouve dans l'Ancien régime avec le délit d'accaparement de biens qui frappe la spéculation sur les " denrées de première nécessité ". Puis I'ancien Code pénal $^{3}$ (article 419) punit tous ceux qui sèment des faits faux ou calomnieux dans le public ou jettent des offres sur le marché à dessein de troubler les cours.

Mais le premier changement réside dans les difficultés auxquelles les États sont de plus en plus confrontés pour réguler et contrôler les acteurs en raison de l'opacité croissante des marchés et de la mondialisation. Le rapport de Christian de Boissieu et al. (2010) sur l'instabilité des marchés agricoles regrette " un déficit de gouvernance mondiale face à l'ampleur des enjeux » dans un contexte où « le déficit de coordination des politiques nationales ne permet pas d'éviter une instabilité qui se diffuse à l'ensemble de la planète ".

Le deuxième changement majeur est lié à la déconnection croissante entre le besoin des marchandises pour elles-mêmes et leur achat. La possession du bien est rarement la cause de la spéculation. Même sur les marchés à terme organisés (futures), les contrats sur les matières premières ne donnent in fine que rarement lieu à livraison, la plupart sont annulés avant l'échéance par une transaction inverse. C'est d'ailleurs ce qui explique l'augmentation du nombre de transactions financières, accentuée encore par le trading à haute fréquence. Avec la financiarisation des marchés agricoles, la spéculation devient alors un but en soi et peut être la cause de l'insécurité alimentaire en nuisant à la formation des prix. 


\section{Inversion de la causalité par rapport au prix}

Selon la morale, si le bien commun détermine le prix, le gain lié à la spéculation est admis. Mais, dans la pratique, l'inversion semble flagrante : la spéculation, sur des mouvements de marché manifestement psychologiques, peut générer des conséquences néfastes sur la formation du prix et sur le bien commun. Ainsi " la théorie des bulles spéculatives montre qu'il est possible de s'écarter durablement des fondamentaux de certains actifs parce que leur prix dépend de l'anticipation de leur valeur future qui comprend une part d'arbitraire. Le prix d'un actif peut alors diverger par rapport à sa valeur d'équilibre, alors même que les investisseurs en sont conscients et savent que la bulle éclatera un jour ou l'autre. Mais l'espérance de gain (si le prix continue à s'accroître) est supérieure à l'espérance de perte (si la bulle éclate) ", constate Jean-Paul Pollin (2004). Dans ce contexte, celui qui parie consciemment sur une bulle spéculative l'encourage de ce fait. Ainsi, lors de la bulle internet, entre 1995 et 2000 , le cours des actions de certaines start-up dans le domaine des nouvelles technologies "capitalisait trois cents ou quatre cents fois les bénéfices annuels l'entreprise fit-elle des pertes!" ", souligne Christian de Boissieu dans le rapport Mancel (2010).

Connus au XVII ${ }^{\mathrm{e}}$ siècle avec la bulle spéculative sur les bulbes de tulipes, ces phénomènes de mimétisme et leur impact sur le prix sont aujourd'hui amplifiés par l'évolution technologique avec le trading algorithmique qui optimise les tâches de prise de décision d'investissement et le trading à haute fréquence qui multiple le nombre d'ordres boursiers. "Alors que le ratio nombre d'ordres sur nombre de transactions était stable et légèrement au-dessus de 1 jusqu'au début des années 2000, il a ensuite fortement augmenté pour atteindre plus de 30. Cela suscite évidemment de sérieuses inquiétudes quant aux possibles manipulations des cours boursiers (quote stuffing, spoofing, etc.). ", alerte Capelle-Blancard (2017).

La formation du prix est désormais dépendante d'une seconde évolution mise en exergue par Lubochinsky dans le rapport Mancel (2010): les «bulles médiatiques ». Les fausses rumeurs, les manipulations ou le cas des falsifications comptables de l'État grec se rapportent toutefois à la question de l'asymétrie d'information abordée dès l'Antiquité.

\section{CONCLUSION}

Cette étude met en évidence que l'inversion de la causalité de la spéculation est la raison de ses effets néfastes aujourd'hui. La spéculation, conséquence d'un besoin réel de marchandises, est parfaitement morale, voire vitale pour la communauté en cas de famine autrefois. Incontestablement le profit est légitime et rémunère notamment le risque encouru. Mais quand la spéculation devient la fin première pour le gain, elle peut être la cause de déséquilibres sur le prix 
des biens, de risques systémiques et d'emballements nocifs pour la collectivité (crise grecque, subprimes). Clairement incriminée, la déconnection actuelle entre les marchés financiers et l'économie réelle éloigne la spéculation de son but initial et moral.

Certes les produits financiers évoluent, mais les préceptes philosophiques restent immuables. Cependant la loi morale a perdu aujourd'hui son influence sur les individus et la devise des boutiquiers romains semble prévaloir : Salve lucrum ! Il est donc du devoir des États d'utiliser l'arme de l'interdiction, en modifiant la loi civile et les structures, pour répondre aux défis nés de la pratique. Mais le contrôle des marchés financiers paraît plus difficile en raison de la mondialisation, des nébuleuses d'intervenants, du développement des produits dérivés, de l'opacité accrue et des milliers d'ordres passés voire annulés en quelques microsecondes. Toutefois le bon fonctionnement du marché semble plus nécessaire encore, eu égard aux dynamiques incontrôlables. Les opérations commerciales contemporaines s'insèrent donc dans un état économique extrêmement complexe qui n'a, à l'évidence, pas pu être soupçonné par un théologien du XIII ${ }^{\mathrm{e}}$ siècle. Dans l'Antiquité et au Moyen Âge, les spéculateurs jouaient, en effet, sur l'augmentation réelle du prix des denrées, non sur des marchés virtuels sans livraison effective des marchandises.

Toutefois les juristes canoniques avaient clairement entrevu l'état social qui est le nôtre et cerné que le juste prix résulte d'une articulation complexe entre justice sociale, rareté, équité et espérance d'utilité. II garantit l'ordre social et doit donc être déterminé par rapport à autrui, non par l'intérêt propre. La place du bien commun de l'ensemble de la société, comme fin ultime, vient alors requalifier les fins particulières du gain du spéculateur. En effet, "l'homme étant essentiellement social, la recherche des fins ne se fait pas dans l'isolement, mais socialement et par référence à des valeurs objectives ", souligne Pierre de Lauzun (2015). Aussi "La responsabilité envers les générations qui suivent est l'une des dimensions essentielles de la conscience morale, et donc d'une économie au service de l'homme. " (Ibid.).

\section{BIBLIOGRAPHIE}

AKERLOF G. (1970), "The Market for "Lemons": Quality Uncertainty and the Market Mecanism ", Quarterly Journal of Economics, vol. 84, pp. 488-500.

ARISTOTE (1848), La politique, traduction de Barthélémy-Saint-Hilaire J., Dumont.

CAPELLE-BLANCARD G. (2017), « À quoi servent les (centaines de milliers de milliards de) transactions boursières ? ", Revue d'économie financière, $\mathrm{n}^{\circ} 127$, pp. 37-58.

CICERON (1933), De officiis, III, 12, traduction de Appuhn C., Paris Garnier.

D'AQUIN T. (1852), Somme théologique, traduction de Drioux C.-J., Belin.

DE BOISSIEU C., JOUYET J.-P. et GUILLON S. (2010), Prévenir et gérer l'instabilité des marchés agricoles, Rapport pour le ministre de l'Agriculture, de l'Alimentation et de la Pêche.

DE GANT H. (1518), Quodlibeta, Paris.

DE LAUZUN P. (2015), La finance peut-elle être au service de l'homme?, Desclée de Brouwer.

DE LA VEGA J. (1688), Confusion de Confusiones, Amsterdam.

ESPINAS A. (1905), "Xénophon: l'économie naturelle et l'impérialisme hellénique ", Revue de métaphysique et de morale, tome 13, $\mathrm{n}^{\circ} 4$, juillet, pp. 545-560.

GARNSEY P. (1996), Famine et approvisionnement dans le monde gréco-romain, Les Belles Lettres. 
GONZALEZ J. (1986), The Lex Irnitana : a New Copy of the Flavian Municipal Law, Journal of Roman Studies, vol. 76, pp. 147-243.

LAPIDUS A. (1986), Le détour de valeur, Economica.

LAUTMAN J. (1969), "La spéculation, facteur d'ordre ou de désordre ? ", Revue française de sociologie, numéro spécial, " Les faits économiques », pp. 608-630.

MANCEL J.-F. (2010), Rapport d'enquête sur les mécanismes de spéculation affectant le fonctionnement des économies, Assemblée Nationale, Rapport n 3034, 14 décembre.

NADEAU R. (2009), "Stratégies de survie et rituels festifs dans le monde gréco-romain", contribution dans l'ouvrage de Bruegel M., Profusion et pénurie - Les hommes face à leurs besoins alimentaires.

PHILOSTRATE (1862), Apollonius de Tyane, traduction de Chassang A., Paris, Librairie académique, Didier et Cie.

POLLIN J.-P. (2004), Finance comportementale et volatilité, Revue d'économie financière, $\mathrm{n}^{\circ} 74$, pp.

139-156.

PROUDHON P.-J. (1857), Manuel du spéculateur à la bourse, Librairie de Garnier frères, 5 ème édition. SPICQ C. (1934), "La moralité du commerce - Les leçons d'une crise au XIII siècle », La Vie Intellectuelle, 25 avril, pp. 460-482.

TODESCHINI G. (2008), Richesse franciscaine : de la pauvreté volontaire à la société de marché, Verdier.

TODESCHINI G. (2017), Les marchands et le temple - La société chrétienne et le cercle vertueux de la richesse du Moyen-Âge à l'époque moderne, Albin Michel, "L'évolution de l'humanité ».

XENOPHON (2008), Économique, Belles Lettres, Classiques en Poche.

${ }^{1}$ Colloque « Histoire de l'économie sans travail. Finances, investissements, spéculation de l'Antiquité à nos jours ", $3^{\mathrm{e}}$ volet : La résolution des conflits, Université de Lille 2, vendredi 18 novembre 2016.

${ }^{2}$ Colloque "Moralité et immoralité des revenus ", $23^{\mathrm{e}}$ colloque du Centre d'éthique économique, Université d'Aix-Marseille, 23 et 24 juin 2016.

${ }^{3}$ L'article 419 a été abrogé en 1994, date d'entrée en vigueur du nouveau Code pénal. 